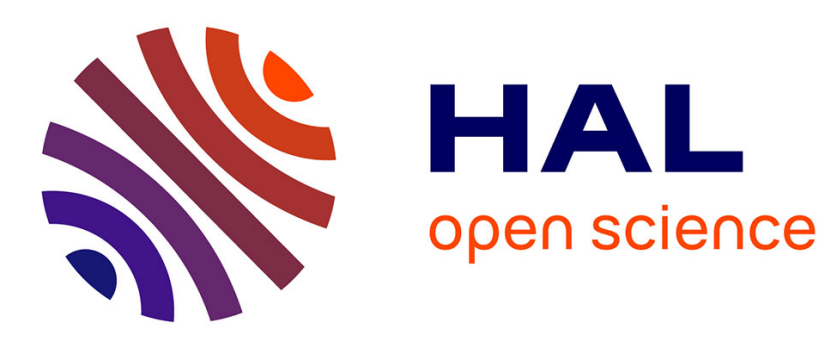

\title{
BL-GARCH model with elliptical distributed innovations
}

\author{
Abdou Kâ Diongue, Dominique Guegan, Rodney C. Wolff
}

\section{To cite this version:}

Abdou Kâ Diongue, Dominique Guegan, Rodney C. Wolff. BL-GARCH model with elliptical distributed innovations. Journal of Statistical Computation and Simulation, 2010, 80 (7), pp.775-791. 10.1080/00949650902773577. halshs-00368340

\section{HAL Id: halshs-00368340 https://shs.hal.science/halshs-00368340}

Submitted on 15 Apr 2009

HAL is a multi-disciplinary open access archive for the deposit and dissemination of scientific research documents, whether they are published or not. The documents may come from teaching and research institutions in France or abroad, or from public or private research centers.
L'archive ouverte pluridisciplinaire HAL, est destinée au dépôt et à la diffusion de documents scientifiques de niveau recherche, publiés ou non, émanant des établissements d'enseignement et de recherche français ou étrangers, des laboratoires publics ou privés. 


\title{
Exact Maximum Likelihood estimator for the BL-GARCH model under elliptical distributed innovations *
}

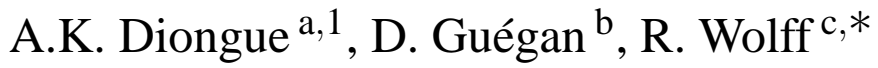 \\ ${ }^{a}$ Université Gaston Berger, UFR SAT, BP 234, Saint-Louis, SENEGAL \\ ${ }^{\mathrm{b}}$ Paris School of Economics, MSE - CES, Université Paris1 Panthéon-Sorbonne, 106 \\ boulevard de l'hopital, 75013 Paris, France \\ 'School of Mathematical Sciences, Queensland University of Technology, GPO Box 2434, \\ Brisbane QLD 4001, Australia
}

\begin{abstract}
We are interested in the parametric class of Bilinear GARCH (BL-GARCH) models which are capable of simultaneously capturing the well known properties of financial retrun series, volatility clustering and leverage effects. Specifically, as it is often observed that the distribution of many financial time series data has heavy tails, heavier than the Normal distribution, we examine, in this paper, the BL-GARCH model in a general setting under some non-normal distributions. We also propose and implement a maximum likelihood estimation (MLE) methodology for parameter estimation. To evaluate the small-sample performance of this method for various models, a Monte Carlo study is conducted. Finally, the capability of within-sample estimation, using the S\&P 500 daily returns, is also studied.
\end{abstract}

Key words: BL-GARCH process, Leverage effects, Maximum likelihood, Monte Carlo method, Volatility clustering.

PACS: 65C05, 82B80, 37M10, 91B84

ऋ This research was supported by an Australian Research Council Discovery Project grant (DP0666266).

* Corresponding author. Tel.: +61 731382080 Fax.: +61 731382310.

Email addresses: abdou.diongue@qut.edu.au (A.K. Diongue), dominique .guegan@univ-paris1.fr (D. Guégan), r.wolff@qut .edu . au (R. Wolff).

1 School of Economics and Finance, Queensland University of Technology, GPO Box 2434, Brisbane QLD 4001, Australia

Preprint submitted to Computational Statistics and Data Analysis

7 March 2008 


\section{Introduction}

The stylized facts of time varying variances have been long recognized in the literature. A popular and prominent tool used to describe this phenomenon is the autoregressive conditional heteroskedasticity (ARCH) model. The ARCH model, developed by Engle (1982) and extended to the GARCH model by Bollerslev (1986), formulates the conditional variance of a random variable as a linear function of its past squared realizations. After the seminal work of Engle (1982), a number of applied works were published to illustrate the usefulness of the GARCH models in economic and financial areas, as in Gouriéroux (1997) and recently Giraitis et al. (2007). Moreover, it is well established in empirical finance that the volatility of many financial assets is asymmetric. Particularly, it has been observed that stock price changes are negatively correlated with changes in volatility which implies that volatility is higher after negative shocks than after positive shocks of the same magnitude: see Black (1976). In the light of this empirical finding, various models with asymmetry in volatility have been proposed. Among others, they include the exponential GARCH (EGARCH) of Nelson (1991), the APARCH model, proposed by Ding et al. (1993), and the BL-GARCH model recently introduced by Storti and Vitale (2003a). The BL-GARCH in Storti and Vitale (2003a) parallel the Gaussian bilinear (BL) model of means dealt with Granger and Andersen (1978) which is used in such fields as signal, environmental studies, demography, economics and finance: see Tong (1990) for a classical review.

The present work focuses on the bilinear model in volatility (BL-GARCH), which has been investigated recently by Storti and Vitale (2003a) for its statistical properties, in that they have derived conditions for the positivity of the conditional variance and for the second order stationarity of the general model. In particluar, for the $\operatorname{BL}-\operatorname{GARCH}(1,1)$, they have provided analytical expressions for the autocorrelation function of the squared process, the unconditional fourth moment and the kurtosis coefficient, together with conditions for the existence of the higher order moments of the process. Furthermore, in order to compare the ability of the model to estimate the volatility of financial time series data with other asymmetric GARCH models, they have applied the model to the continuously compounded returns S\&P 500 index. However, parameter estimation of the BL-GARCH model remains problematic. Indeed, to deal with the parameter estimation for the BL-GARCH model, Storti and Vitale (2003b) used an indirect maximum likelihood procedure based on the EM algorithm. Despite the fact that the EM algorithm has become a very popular computational method in statistics, this approach presents some limitations: slow numerical convergence, the convergence to a maximum likelihood estimator depending on a judicious choice of the starting value, and the underlying assumption of normally distributed data. These would seem to limit its applicability and also there is the issue of the non-existence of a measure of the standard errors of the estimates. In addition, while, BL-GARCH models adequately capture volatil- 
ity clustering and asymmetry, coupled with the auxiliary assumption of normally distributed errors, the model does not fully capture the very fat-tailed property of high frequency financial time series, often observed in exchange rates, stock returns and commodity returns, as in Baillie and Bollerslev (1991), McAleer (2005), Zivot and Wang (2005) and references therein. Actually, several alternative distributions have been proposed in the literature to model such excess kurtosis in the conditional distribution of returns better including the Student-t of Bollerslev (1987) and the generalized error distribution (GED) of Nelson (1991). Hansen (1994) introduced the use of an asymmetric Student-t type distribution to capture the skewness property well. Since then, other articles have studied different skew Student-t type distributions for financial and other applications: Azzalini and Capitanio (2003), Jones and Faddy (2003) and Patton (2004). All of these distributions try to account for substantial departure from the Normal distribution, specifically large kurtosis evident in the empirical distribution of the returns, more peakedness and with fatter tails than the Normal distribution. However, while large kurtosis of the returns is a well-established fact, the situation is much more obscure with regard to the symmetry of the distribution. Indeed, testing the symmetry of unconditional distributions of eight international stock market returns, Peiró (1999) concluded that, under the alternative of non-normal distributions, the symmetry of the returns cannot be rejected for most markets. Furthermore, investigating distribution-free methods, he finds that in most markets, daily financial returns are symmetric or, at least, do not present strong evidence of skewness.

The main purpose of the present study is first, following the empirical evidence of leptokurtosis in financial returns, to present an extension of the BL-GARCH with Normal distribution to other alternative non-Gaussian distributions. We therefore consider the elliptical distribution family, as it is rich enough to include the Normal, Student-t, GED and many others, Bingham and Kiesel (2002). Secondly, given that the maximum likelihood method is widely used when dealing with parameter estimation for GARCH models, Li et al. (2002) or McNeil et al. (2005), we also investigate the problem of estimating parameters of the BL-GARCH model using MLE and examine its finite sample properties in a series of Monte Carlo simulation experiments. This is completely new, in that Storti and Vitale (2003b) only give an empirical approach for the EM method.

The paper is organized as follows. In Section 2, the BL-GARCH model is presented with some important properties concerning conditions for the conditional variance to be finite, as well as for strictly stationarity and ergodicity solutions. In Section 3, we present the MLE method under elliptical distributions, such as the Normal, Student-t and GED. We also provide the score functions as well as the Hessian matrices for these models. Section 4 studies the performance of the estimation procedure through Monte Carlo simulations. Section 5 presents the data and contains the main empirical findings along with the goodness-of-fit tests, while Section 6 
provides concluding remarks.

\section{BL-GARCH model and its specification}

Over the past two decades, enormous efforts have been devoted to modelling and forecasting the volatility of stock returns and other financial time series. Seminal work in this area of research can be attributed to Engle (1982), who introduced the standard autoregressive conditional heteroscedasticity (ARCH) model. Since then, further extensions have been investigated including the GARCH model proposed by Bollerslev (1986), and asymmetric GARCH models such as the EGARCH model of Nelson (1991), the APARCH model of Ding et al. (1993), and recently the BL-GARCH model, with which we deal in this paper, studied by Storti and Vitale (2003a).

\subsection{The BL-GARCH model}

Let $S_{t}$ denote the price of an asset at time $t, y_{t}=\log \left(S_{t} / S_{t-1}\right)$ the continuously compounded return series and $\mu_{t}=E\left(y_{t} \mid \Psi_{t-1}\right)$ the conditional mean given an increasing sequence of $\sigma$-fields $\Psi_{t-1}$ generated by $\left(y_{t-1}, y_{t-2}, \cdots\right)$. Assume that the series of interest, $y_{t}$, is given by, for $t=1, \cdots, n$,

$$
\begin{gathered}
y_{t}=\mu_{t}+u_{t}, \\
u_{t}=h_{t} \varepsilon_{t}, \\
h_{t}^{2}=a_{0}+\sum_{i=1}^{p} a_{i} u_{t-i}^{2}+\sum_{j=1}^{q} b_{j} h_{t-j}^{2}+\sum_{k=1}^{r} c_{k} h_{t-k} u_{t-k},
\end{gathered}
$$

where $p, q, r$ are non-negative integers with $r=\min (p, q), h_{t}^{2}$ the conditional variance of the process $\left(u_{t}\right)_{t}$ given the $\sigma$-fields $\Psi_{t-1}$ and $\varepsilon_{t} \sim$ i.i.d. $D(0,1)$ with $D($. a probability density function with mean 0 and unit variance (it will be defined in Section 3). The model (1b)-(1c) is more general than the standard GARCH model of Bollerslev (1986) in the sense that it allows innovations of different signs to have a different impact on volatility and allows larger shocks to have a larger impact on volatility than the standard GARCH model $^{2}$.

2 Changes in stock prices tend to be negatively correlated related to changes in volatility, Black (1976). Specifically, lagged unexpected declines in prices (bad news) increase current volatility more than to lagged unexpected increases in prices (good news). This asymmetry in stock returns volatility has been termed the "leverage-effect". 


\subsection{Regularity conditions}

\subsubsection{Positivity conditions}

Sufficient conditions for the positivity of the conditional variance $h_{t}^{2}$ have been provided in the paper by Storti and Vitale (2003a). To this end, let

$$
R=\left[\begin{array}{ccccc}
a_{0} & 0 & \cdots & \cdots & 0 \\
0 & R_{1} & 0 & \cdots & 0 \\
0 & \ddots & \ddots & & 0 \\
\vdots & & \ddots & \ddots & \vdots \\
0 & \cdots & \cdots & 0 & R_{r}
\end{array}\right] \text { and } S=\left[\begin{array}{ccccc}
s_{1} & \cdots & 0 & \cdots & 0 \\
0 & \ddots & 0 & \cdots & 0 \\
\vdots & \ddots & \ddots & \ddots & \vdots \\
0 & & \ddots & s_{m-1} & 0 \\
0 & \cdots & \cdots & 0 & s_{m}
\end{array}\right]
$$

where, for $i=1, \cdots, r, R_{i}=\left[\begin{array}{cc}a_{i} & \frac{1}{2} c_{i} \\ \frac{1}{2} c_{i} & b_{i}\end{array}\right]$ and, for $i=1, \cdots, m$, with $m=\max (p, q)-$ $r$, we set $s_{i}=a_{r+i}$, if $p>q$, or $s_{i}=b_{r+i}$, if $p<q$. Hence, if $a_{0}>0$, a sufficient condition for the positivity of $h_{t}^{2}$ is given by $s_{i}>0(i=1, \cdots, m)$ and all matrices $R_{i}$ $(i=1, \cdots, r)$ being positive semi-definite. Furthermore, for the $\operatorname{BL}-\operatorname{GARCH}(1,1)$ model, the positive definiteness of the matrix $R_{1}$ is a necessary and sufficient condition for the positivity of $h_{t}^{2}$.

\subsubsection{Covariance stationarity conditions}

Storti and Vitale (2003a) show also that the stationarity condition of the BL-GARCH model is analogous to that stated for GARCH models by Bollerslev (1986). Hence, the shocks $h_{t} \varepsilon_{t}$ are covariance stationary and non-degenerate if

(i) $\operatorname{var}\left(\varepsilon_{t}\right)<\infty$;

(ii) $a_{0}>0$; and

(iii) $\sum_{i=1}^{p} a_{i}+\sum_{j=1}^{q} b_{j}<1$.

Under the assumption that the innovations are elliptically distributed with zero mean and unit variance, the first condition is obviously satisfied by construction. The second and third conditions can either be enforced during estimation or verified afterwards. 


\section{Maximum likelihood approach}

Estimation of conditional volatility models are typically performed by an MLE procedure, as in Bollerslev and Wooldridge (1992). The conditional likelihood function is given by

$$
\mathscr{L}(\omega)=\mathscr{L}\left(y_{1}, \cdots, y_{n} \mid \omega\right)=\prod_{t=1}^{n} g\left(y_{t}, \mu_{t}(\alpha), h_{t}(\omega)\right),
$$

where $g\left(y_{t}, \mu_{t}(\alpha), h_{t}(\omega)\right)$ denotes the conditional density function for the random variables $y_{t}$ with mean $\mu_{t}$ and standard deviation $h_{t}$, and $\omega=(\alpha, \theta)$ is the parameter vector to be estimated, and where $\alpha$ corresponds to the set of parameters in the condtional mean assumed, in what follows, to be an $\operatorname{ARMA}(k, l)$ model and $\theta=$ $\left(a_{0}, a_{1}, \cdots, a_{p}, b_{1}, \cdots, b_{q}, c_{1}, \cdots, c_{r}\right)$. Following Storti and Vitale (2003a), we make the following theoretical assumption about the parameter space, $\Omega$, and the true parameter vector, $\omega_{0}=\left(\alpha_{0}, \theta_{0}\right)^{\prime}$ :

Assumption 1: the parameter $\omega_{0} \in \Omega \subseteq \mathbb{R}^{k+l+p+q+r+1}$ is in the interior of $\Omega$, a compact parameter space. Specifically for any vector $\omega \in \Omega$, assume that

(1) the AR and MA polynomials have no common roots and that all their roots lie outside the unit circle;

(2) $a_{0}>0, a_{1}, \cdots, a_{p} \geq 0$, and $b_{1}, \cdots, b_{q} \geq 0$;

(3) $c_{i}^{2}<4 a_{i} b_{i}$, for $i=1, \cdots, r$; and

(4) $\sum_{i=1}^{p} a_{i}+\sum_{i=1}^{q} b_{i}<1$.

Thus, estimation proceeds by maximising, under the Assumption $1, L(\omega)=\log (\mathscr{L}(\omega))$, where pre-sample values of $h_{t}^{2}$ are set to the unconditional sample variance.

Since it may be expected that excess kurtosis and skewness displayed by the residuals of conditional heteroscedasticity models will be reduced when a more appropriate distribution is used, we consider in this study the three most typical elliptical normalized distributions that have been applied so far: the Normal, Student-t and GED distributions.

\subsection{Normal distribution}

The Normal distribution is the most widely used when estimating GARCH models. If we assume that the innovations $\left(\varepsilon_{t}\right)_{t \in \mathbb{Z}}$ have a conditional Gaussian distribution 
then the conditional log-likelihood function associated to $y_{t}, u_{t}=y_{t}-\mu_{t}$, is given by

$$
L(\omega)=-\frac{n}{2} \log (2 \pi)-\frac{1}{2} \sum_{t=1}^{n}\left[\log \left(h_{t}^{2}\right)+\frac{u_{t}^{2}}{h_{t}^{2}}\right],
$$

where $n$ is the number of observations. To obtain an analytical or numerical solution for the MLE, we need the first-order derivative and to solve the equation $\partial L(\omega) / \partial \omega=0$. Taking the differential of $L(\omega)$ in (4) with respect to the full set of parameter $\omega$ yields

$$
\frac{\partial L(\omega)}{\partial \omega}=\sum_{t=1}^{n} \frac{u_{t}}{h_{t}^{2}} \frac{\partial \mu_{t}}{\partial \omega}+\frac{1}{2} \sum_{t=1}^{n} \frac{1}{h_{t}^{2}}\left(\frac{u_{t}^{2}}{h_{t}^{2}}-1\right) \frac{\partial h_{t}^{2}}{\partial \omega} .
$$

The Hessian matrix is given by

$$
\begin{aligned}
\frac{\partial^{2} L(\omega)}{\partial \omega \partial \omega^{\prime}}= & -\sum_{t=1}^{n} \frac{1}{h_{t}^{2}} \frac{\partial \mu_{t}}{\partial \omega} \frac{\partial \mu_{t}}{\partial \omega^{\prime}}-\sum_{t=1}^{n} \frac{u_{t}}{h_{t}^{4}} \frac{\partial \mu_{t}}{\partial \omega} \frac{\partial h_{t}^{2}}{\partial \omega^{\prime}} \\
& -\sum_{t=1}^{n} \frac{u_{t}}{h_{t}^{4}} \frac{\partial \mu_{t}}{\partial \omega} \frac{\partial h_{t}^{2}}{\partial \omega^{\prime}}+\sum_{t=1}^{n} \frac{1}{h_{t}^{4}}\left(\frac{1}{2}-\frac{u_{t}^{2}}{h_{t}^{2}}\right) \frac{\partial h_{t}^{2}}{\partial \omega} \frac{\partial h_{t}^{2}}{\partial \omega^{\prime}} .
\end{aligned}
$$

\subsection{Student-t distribution}

Now, if we assume that the innovations $\left(\varepsilon_{t}\right)_{t \in \mathbb{Z}}$ have a conditional Student-t distribution with $v$ degrees of freedom, then the MLE estimator $\hat{\omega}_{n}$ maximises the $\log$-likelihood function $L(\omega)$ given by

$$
\begin{aligned}
L(\omega)= & n\left[\log \Gamma\left(\frac{v+1}{2}\right)-\log \Gamma\left(\frac{v}{2}\right)-\frac{1}{2} \log \pi(v-2)\right] \\
& -\frac{1}{2} \sum_{t=1}^{n}\left\{\log \left(h_{t}^{2}\right)+(v+1) \log \left[1+\frac{u_{t}^{2}}{h_{t}^{2}(v-2)}\right]\right\},
\end{aligned}
$$

where $2<v \leq \infty$ and $\Gamma$ is the Euler gamma function defined by $\Gamma(x)=\int_{0}^{\infty} t^{x-1} e^{-t} d t$. When $v \rightarrow \infty$, we have the Normal distribution, so that the smaller the value of $v$ the fatter the tails. The score function is given by

$$
\begin{aligned}
\frac{\partial L(\omega)}{\partial \omega}= & \sum_{t=1}^{n}\left[\frac{v+1}{v-2} \frac{u_{t}}{h_{t}^{2}}\left(1+\frac{u_{t}^{2}}{h_{t}^{2}(v-2)}\right)^{-1}\right] \frac{\partial \mu_{t}}{\partial \omega} \\
& +\frac{1}{2} \sum_{t=1}^{n}\left[\frac{v+1}{v-2} \frac{u_{t}^{2}}{h_{t}^{2}}\left(1+\frac{u_{t}^{2}}{h_{t}^{2}(v-2)}\right)^{-1}-1\right] \frac{1}{h_{t}^{2}} \frac{\partial h_{t}^{2}}{\partial \omega}
\end{aligned}
$$


and the Hessian matrix is given by

$$
\begin{aligned}
\frac{\partial^{2} L(\omega)}{\partial \omega \partial \omega^{\prime}}= & \frac{v+1}{v-2} \sum_{t=1}^{n}\left(1+\frac{u_{t}^{2}}{(v-2) h_{t}^{2}}\right)^{-1} \frac{u_{t}}{h_{t}^{2}}\left[\frac{2}{v-2}\left(1+\frac{u_{t}^{2}}{(v-2) h_{t}^{2}}\right)^{-1} \frac{u_{t}}{h_{t}^{2}}-1\right] \frac{\partial \mu_{t}}{\partial \omega} \frac{\partial \mu_{t}}{\partial \omega^{\prime}} \\
& +\frac{v+1}{v-2} \sum_{t=1}^{n}\left(1+\frac{u_{t}^{2}}{(v-2) h_{t}^{2}}\right)^{-1} \frac{u_{t}}{h_{t}^{4}}\left[\frac{1}{v-2}\left(1+\frac{u_{t}^{2}}{(v-2) h_{t}^{2}}\right)^{-1} \frac{u_{t}^{2}}{h_{t}^{2}}-1\right] \frac{\partial \mu_{t}}{\partial \omega} \frac{\partial h_{t}^{2}}{\partial \omega^{\prime}} \\
& +\frac{v+1}{v-2} \sum_{t=1}^{n}\left(1+\frac{u_{t}^{2}}{(v-2) h_{t}^{2}}\right)^{-1} \frac{u_{t}}{h_{t}^{4}}\left[\frac{1}{v-2}\left(1+\frac{u_{t}^{2}}{(v-2) h_{t}^{2}}\right)^{-1} \frac{u_{t}^{2}}{h_{t}^{2}}-1\right] \frac{\partial h_{t}^{2}}{\partial \omega} \frac{\partial \mu_{t}}{\partial \omega^{\prime}} \\
& +\frac{1}{2} \sum_{t=1}^{n} \frac{1}{h_{t}^{4}}\left[1+\frac{(v+1) u_{t}^{2}}{(v-2) h_{t}^{2}}\left(1+\frac{u_{t}^{2}}{(v-2) h_{t}^{2}}\right)^{-1}\right] \\
& \left.+\frac{u_{t}^{2}}{(v-2) h_{t}^{2}}\left(1+\frac{u_{t}^{2}}{(v-2) h_{t}^{2}}\right)^{-1}-2\right] \frac{\partial h_{t}^{2}}{\partial \omega} \frac{\partial h_{t}^{2}}{\partial \omega^{\prime}} .
\end{aligned}
$$

\subsection{GED distribution}

Knowing that skewness and kurtosis are important in financial applications, Nelson (1991) suggested to consider the family of GEDs. The probability density function of a normalized GED random variable is given by

$$
f(x)=\frac{v 2^{-\left(1+\frac{1}{v}\right)}}{\lambda_{v} \Gamma\left(\frac{1}{v}\right)} e^{-\frac{1}{2}\left|\frac{x}{\lambda_{v}}\right|^{v}},-\infty<x<\infty,
$$

with $\lambda_{v}=\sqrt{2^{-2 / v} \Gamma(1 / v) / \Gamma(3 / v)}$ and $0<v<\infty$ is the tail-thickness parameter. The GED includes the Gaussian distribution $(v=2)$ as a special case, along with many other distributions, some more fat-tailed than the Gaussian (e.g., the double exponential distribution corresponding to $v=1$ ) and some more thin-tailed (e.g., the Uniform distribution on the interval $[-\sqrt{3}, \sqrt{3}]$ when $v \rightarrow \infty)$. The GED loglikelihood function is given by

$$
\begin{aligned}
L(\omega)= & {\left[\log \left(\frac{v}{\lambda_{v}}\right)-\left(1+\frac{1}{v}\right) \log (2)-\log \Gamma\left(\frac{1}{v}\right)\right] } \\
& -\frac{1}{2} \sum_{t=1}^{n}\left[\log \left(h_{t}^{2}\right)+h_{t}^{-v}\left|\frac{u_{t}}{\lambda_{v}}\right|^{v}\right] .
\end{aligned}
$$

In this case, the score function and the Hessian matrix, respectively, are given by

$$
\frac{\partial L(\omega)}{\partial \omega}=\frac{1}{2} \sum_{t=1}^{n} \frac{v}{\left|\lambda_{v}\right|}\left(\frac{u_{t}}{h_{t}}\right)^{v} \frac{1}{u_{t}} \frac{\partial \mu_{t}}{\partial \omega}+\frac{1}{2} \sum_{t=1}^{n} \frac{1}{h_{t}^{2}}\left[\frac{1}{2} \frac{l}{\left|\lambda_{v}\right|^{v}}\left(\frac{u_{t}}{h_{t}}\right)^{v}-1\right] \frac{\partial h_{t}^{2}}{\partial \omega},
$$


and

$$
\begin{aligned}
\frac{\partial^{2} L(\omega)}{\partial \omega \partial \omega^{\prime}}= & -\frac{v}{\left|\lambda_{v}\right|^{v}}\left(\frac{v-3}{2}\right) \sum_{t=1}^{n} \frac{1}{h_{t}^{2}}\left(\frac{u_{t}^{2}}{h_{t}^{2}}\right)^{\frac{v}{2}-1} \frac{\partial \mu_{t}}{\partial \omega} \frac{\partial \mu_{t}}{\partial \omega^{\prime}} \\
& -\frac{1}{2} \frac{v}{\left|\lambda_{v}\right|^{v}} \sum_{t=1}^{n} \frac{u_{t}}{h_{t}^{4}}\left(\frac{u_{t}^{2}}{h_{t}^{2}}\right)^{\frac{v}{2}-1}\left[1+\left(\frac{v}{2}-1\right) \frac{u_{t}^{2}}{h_{t}^{2}}\right] \\
& -\frac{1}{2} \frac{v}{\left|\lambda_{v}\right|^{v}} \sum_{t=1}^{n} \frac{u_{t}}{h_{t}^{4}}\left(\frac{u_{t}^{2}}{h_{t}^{2}}\right)^{\frac{v}{2}-1}\left[1+\left(\frac{v}{2}-1\right) \frac{u_{t}^{2}}{h_{t}^{2}}\right] \\
& -\frac{1}{2} \sum_{t=1}^{n}\left[\frac{1}{4} \frac{v(v+2)}{\left|\lambda_{v}\right|^{v}}\left(\frac{u_{t}}{h_{t}}\right)^{v}-1\right] \frac{1}{h_{t}^{4}} \frac{\partial h_{t}^{2}}{\partial \omega} \frac{\partial h_{t}^{2}}{\partial \omega^{\prime}}
\end{aligned}
$$

Equations (5) through (13) require the computation of $\partial h_{t}^{2} / \partial \omega$. This is given by

$$
\begin{aligned}
\frac{\partial h_{t}^{2}}{\partial \omega}= & \left(1, u_{t-1}^{2}, \cdots, u_{t-p}^{2}, h_{t-1}^{2}, \cdots, h_{t-q}^{2}, h_{t-1} u_{t-1}, \cdots, h_{t-r} u_{t-r}\right) \\
& +2 \sum_{i=1}^{p} a_{i} u_{t-1} \frac{\partial u_{t-i}}{\partial \omega}+\sum_{j=1}^{q} b_{j} \frac{\partial h_{t-j}^{2}}{\partial \omega} \\
& +\sum_{k=1}^{r} c_{k}\left(h_{t-k} \frac{\partial u_{t-k}}{\partial \omega}+\frac{1}{2} \frac{u_{t-k}}{h_{t-k}} \frac{\partial h_{t-k}^{2}}{\partial \omega}\right) .
\end{aligned}
$$

The use and analysis of the MLE method for the estimation problem is classical. A main attraction is the general feature that maximum likelihood estimators achieve optimal accuracy, in that they are asymptotically consistent (in data length $n$ ), and achieve the Cramér-Rao lower bound on estimate variability. Despite these advantages, an important obstacle to employing the method is the difficulty of computing a value $\hat{\omega}_{M L E}$ that satisfies Assumption 1. In the next section, the practical applicability and small sample performance of the MLE procedure for BL-GARCH processes are studied by Monte Carlo simulations.

\section{Monte Carlo experiments}

To our knowledge, no results exist on the properties of these estimators when we observe a finite segment of (1a)-(1c). Thus, we have designed and executed a Monte Carlo experiment using the different distributions described in the previous section as data generating processes, with the aim of analyzing the sampling properties of the exact MLE estimators of the parameter vector $\omega$ for the BL-GARCH model. Through the Monte Carlo experiment, the model considered for $u_{t}=y_{t}-\mu_{t}$ is a 
$\operatorname{BL}-\operatorname{GARCH}(1,1)$ given by

$$
\begin{gathered}
u_{t}=h_{t} \varepsilon_{t}, \text { for } t=1, \cdots, n, \\
h_{t}^{2}=a_{0}+a_{1} u_{t-1}^{2}+b_{1} h_{t-1}^{2}+c_{1} h_{t-1} u_{t-1},
\end{gathered}
$$

with $\varepsilon_{t}$ is a standard Normal, Student-t or GED random variable and $n=500,1000$, 2000 and 3000. Two cases are studied in the simulation experiments. In the first case, the conditional mean, $\mu_{t}$, is taken equal to zero while in the second one we assume that it follows an $\operatorname{AR}(1)$ model $^{3}$. The data generating processes are summarized in Table 1 with the first three lines corresponding to the case $\mu_{t}=0$ and the last line $\mu_{t}=\alpha_{0}+\alpha_{1} y_{t-1}$. The data generating processes, correponding to the case $\mu_{t}=0$, are as in Storti and Vitale (2003a). Throughout the simulations, we consider a Student-t with five degrees of freedom, the same as used in Tsay (2002). Thus, the first four moments of the conditional density exist. For the GED distribution, we assume that the tail-thickness parameter is equal to three.

Table 1

Data generating processes (DGPs)

\begin{tabular}{lcccccc}
\hline DGP & $\alpha_{0}$ & $\alpha_{1}$ & $a_{0}$ & $a_{1}$ & $b_{1}$ & $c_{1}$ \\
\hline Model 1 & 0 & 0 & 0.01 & 0.09 & 0.9 & 0.15 \\
Model 2 & 0 & 0 & 0.05 & 0.05 & 0.9 & 0.25 \\
Model 3 & 0 & 0 & 0.2 & 0.05 & 0.75 & 0.35 \\
Model 4 & 0.01 & 0.2 & 0.01 & 0.09 & 0.9 & 0.15 \\
\hline
\end{tabular}

Tables 2-4 list the Monte Carlo mean, mean absolute error (MAE) and root mean square error (RMSE) for the parameter vector $\omega$ across $M=1000$ Monte Carlo simulations. The simulation algorithm generates $n+500$ observations for each series, saving only the last $n$. This operation is performed in order to avoid dependence on initial values. The calculations were carried out in Matlab on a Pentium IV CPU $3.00 \mathrm{GHz}$ computer. Inspection of Table 2, corresponding to the Normal case, reveals that, for both sample sizes, the averages obtained from the exact MLE are close to the true parameter values. The corresponding MAE and RMSE are very small indicating that estimators are asymptotically unbiased and consistent, respectively. Tables 3 and 4 present the estimated results from a non-Gaussian BLGARCH model. We read, from these tables, that the averages of the parameter estimates are close to the true values under each of the underlying non-Normal error terms. The MAE as well as the RMSE are quite small and decrease when the sample sizes increase. Finally, Table 5 summarizes the results from the AR(1)-BL$\operatorname{GARCH}(1,1)$. Results reveal that parameter estimates are satisfactory in that the

$\overline{3}$ Other simulation results are available upon request. 
MAE and also RMSE are small. We can also remark that, in general, the estimators of the autoregressive models seem not to be affected by, the presence of the BLGARCH errors. In addition, the method seems applicable, even if the sample size is less than 100, due to the fact that, in general, the true values are contained with \pm 2 standard deviations of MLE's estimates: see Figures 1-4.

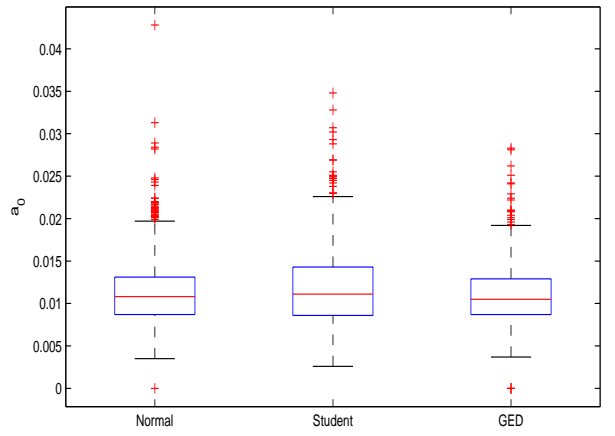

Fig. 1. Boxplot of the parameter $a_{0}$ of Model 1 under Normal, Student-t and GED distributions $(n=1000)$

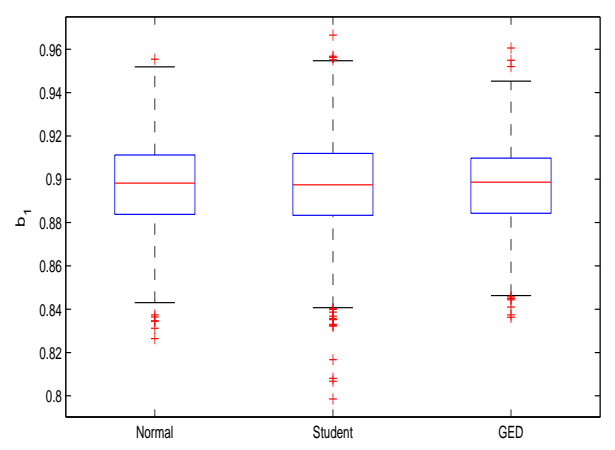

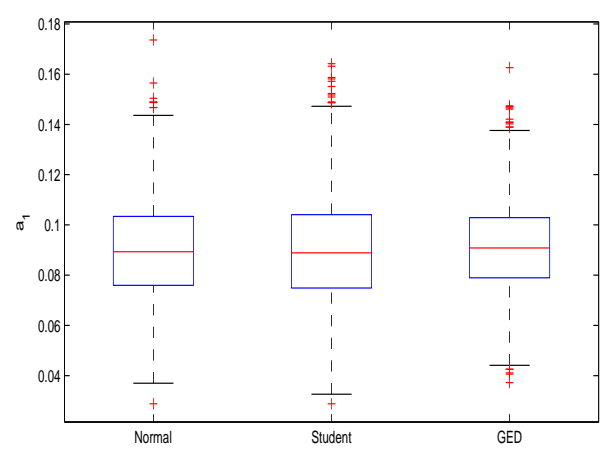

Fig. 2. Boxplot of the parameter $a_{1}$ of Model 1 under Normal, Student-t and GED distributions $(n=1000)$

Fig. 3. Boxplot of the parameter $b_{1}$ of ModelFig. 4. Boxplot of the parameter $c_{1}$ of Model 1 under Normal, Student-t and GED distribu-1 under Normal, Student-t and GED distributions $(n=1000)$

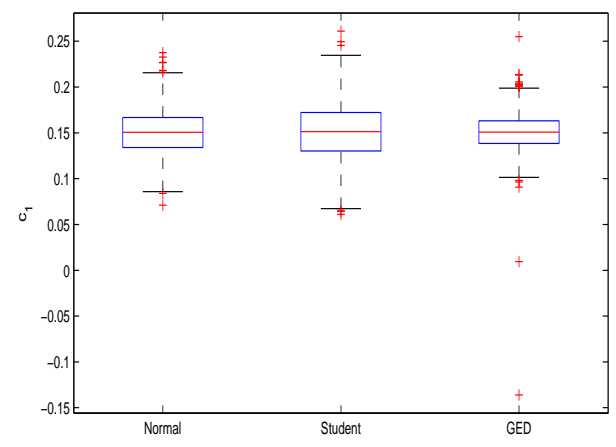




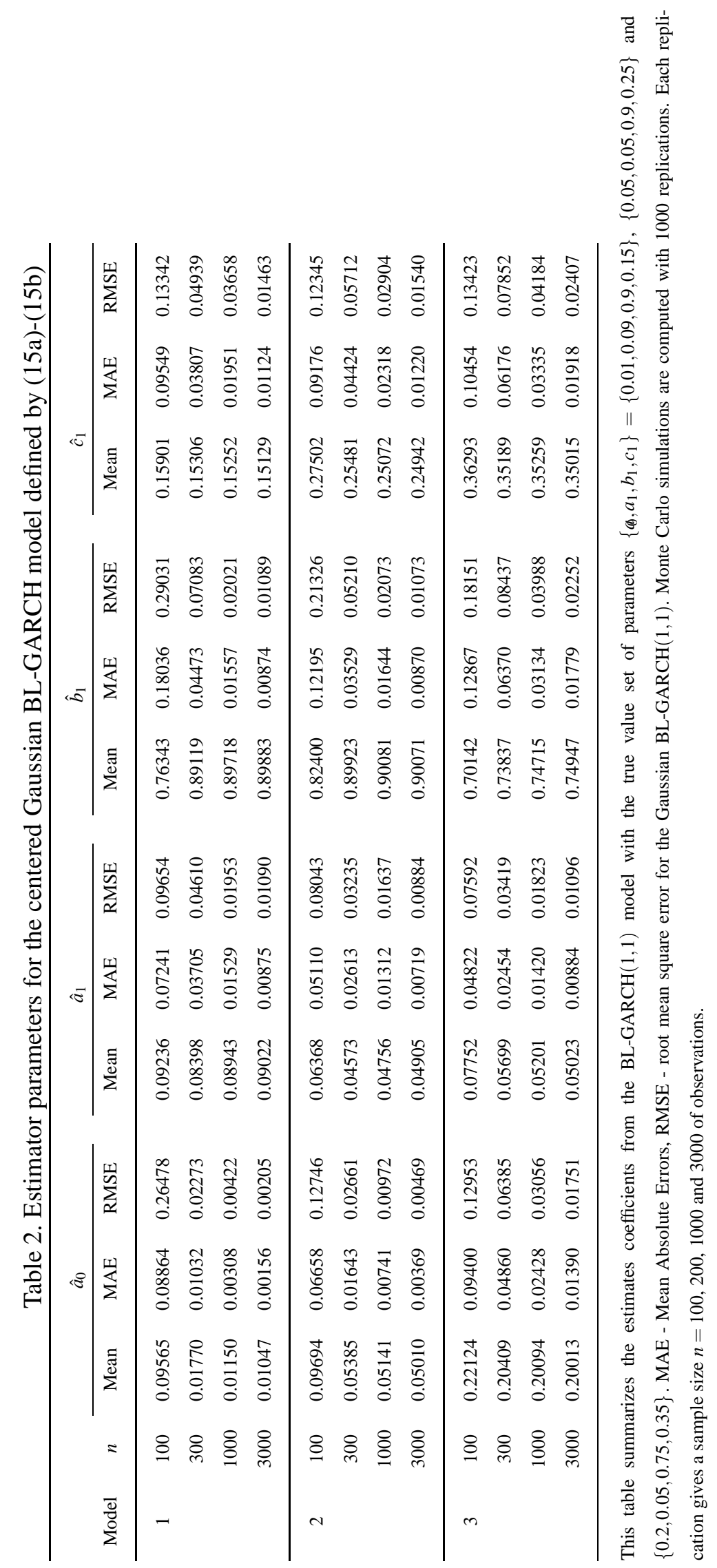




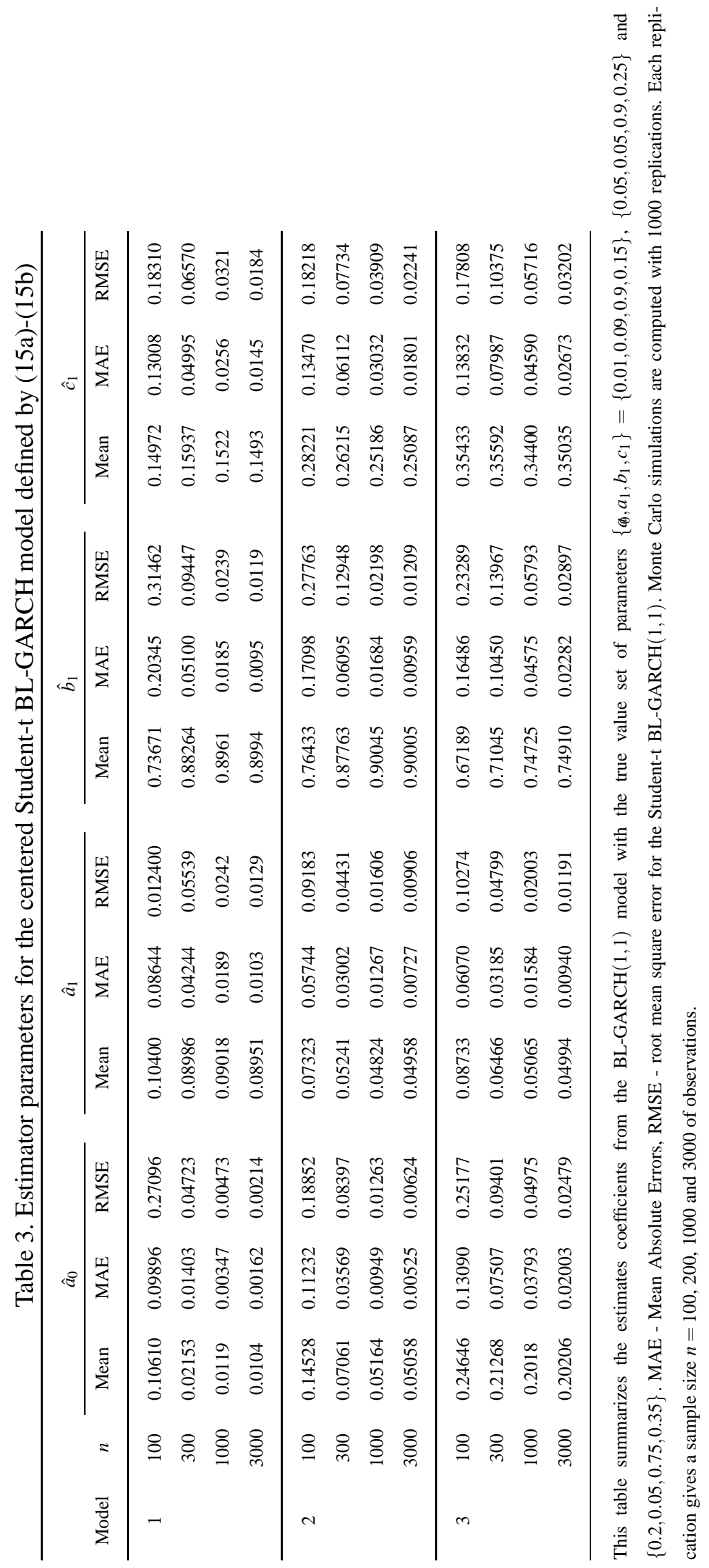




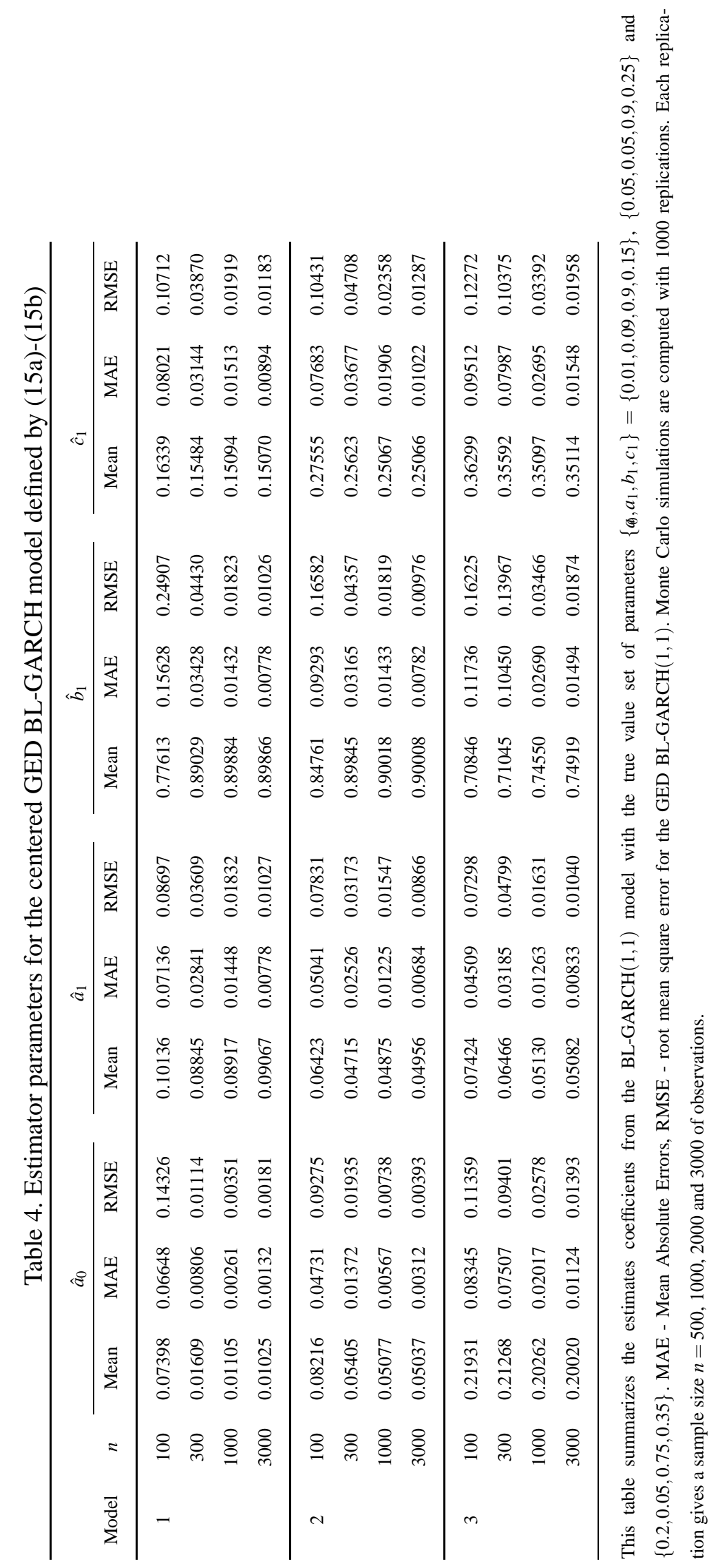


Table 5

Estimator parameters for the centered AR-BL-GARCH model defined by (15a)-(15b)

\begin{tabular}{|c|c|c|c|c|c|c|c|}
\hline Distribution & $\mathrm{n}$ & $\hat{\alpha}_{0}$ & $\hat{\alpha}_{1}$ & $\hat{a}_{0}$ & $\hat{a}_{1}$ & $\hat{b}_{1}$ & $\hat{c}_{1}$ \\
\hline \multirow[t]{12}{*}{ Normal } & 100 & 0.0257 & 0.18447 & 0.10767 & 0.09233 & 0.78056 & 0.16659 \\
\hline & & $(0.06246)$ & $(0.08631)$ & $(0.08060)$ & $(0.07867)$ & $(0.17161)$ & $(0.09643)$ \\
\hline & & [0.09254] & {$[0.08737]$} & [0.24972] & [0.10633] & [0.28043] & [0.13219] \\
\hline & 300 & 0.01897 & 0.19377 & 0.01874 & 0.0838 & 0.88978 & 0.15629 \\
\hline & & $(0.03242)$ & $(0.04678)$ & $(0.01141)$ & $(0.03729)$ & $(0.04555)$ & (0.03919) \\
\hline & & [0.04338] & [0.05949] & {$[0.02728]$} & [0.04697] & {$[0.07751]$} & [0.05055] \\
\hline & 1000 & 0.01103 & 0.19871 & 0.01142 & 0.08847 & 0.89841 & 0.15103 \\
\hline & & $(0.01624)$ & $(0.02544)$ & $(0.00322)$ & $(0.01555)$ & $(0.01603)$ & $(0.01932)$ \\
\hline & & [0.02069] & [0.03167] & {$[0.00441]$} & [0.01995] & [0.02064] & [0.02422] \\
\hline & 3000 & 0.01117 & 0.19905 & 0.01042 & 0.08998 & 0.89941 & 0.15086 \\
\hline & & $(0.00911)$ & $(0.01477)$ & $(0.00156)$ & $(0.0088)$ & $(0.00871)$ & (0.01129) \\
\hline & & [0.01189] & [0.0187] & [0.00209] & [0.01134] & [0.01117] & [0.01444] \\
\hline \multirow[t]{12}{*}{ Student- $\mathrm{t}$} & 100 & 0.0209 & 0.18876 & 0.08852 & 0.09884 & 0.7457 & 0.15474 \\
\hline & & $(0.05193)$ & $(0.08171)$ & $(0.08201)$ & $(0.08648)$ & $(0.20145)$ & $(0.13304)$ \\
\hline & & [0.07803] & [0.10338] & [0.23158] & [0.11957] & [0.32115] & [0.17796] \\
\hline & 300 & 0.01472 & 0.19351 & 0.02124 & 0.08836 & 0.87681 & 0.15205 \\
\hline & & $(0.02583)$ & $(0.04575)$ & $(0.01389)$ & $(0.04023)$ & $(0.05523)$ & $(0.05247)$ \\
\hline & & [0.03494] & [0.05758] & [0.03351] & [0.05389] & [0.10593] & [0.06717] \\
\hline & 1000 & 0.01123 & 0.19838 & 0.01206 & 0.09095 & 0.89505 & 0.15105 \\
\hline & & $(0.01378)$ & $(0.02474)$ & $(0.0036)$ & $(0.01713)$ & $(0.01776)$ & $(0.02524)$ \\
\hline & & {$[0.01737]$} & {$[0.031]$} & [0.00523] & [0.02215] & {$[0.0227]$} & [0.03156] \\
\hline & 3000 & 0.01053 & 0.19941 & 0.01054 & 0.09075 & 0.89861 & 0.15111 \\
\hline & & $(0.00794)$ & $(0.01389)$ & $(0.0018)$ & $(0.00992)$ & $(0.00957)$ & $(0.0151)$ \\
\hline & & [0.01013] & {$[0.01748]$} & {$[0.00278]$} & [0.01286] & {$[0.01323]$} & [0.01999] \\
\hline \multirow[t]{12}{*}{ GED } & 100 & 0.01715 & 0.18247 & 0.09296 & 0.09692 & 0.77744 & 0.16364 \\
\hline & & $(0.06385)$ & $(0.08462)$ & $(0.08650)$ & $(0.07818)$ & (0.17089) & (0.08186) \\
\hline & & [0.09838] & [0.10735] & [0.10735] & [0.10405] & [0.28519] & [0.11175] \\
\hline & 300 & 0.01680 & 0.19660 & 0.01619 & 0.08756 & 0.89222 & 0.15398 \\
\hline & & $(0.03025)$ & $(0.04396)$ & $(0.00882)$ & $(0.03510)$ & $(0.03925)$ & $(0.0323)$ \\
\hline & & [0.04067] & [0.05591] & [0.02660] & [0.04534] & {$[0.06616]$} & [0.04133] \\
\hline & 1000 & 0.01253 & 0.19973 & 0.01098 & 0.0914 & 0.89718 & 0.15228 \\
\hline & & $(0.0163)$ & $(0.02493)$ & $(0.0028)$ & $(0.01539)$ & $(0.01545)$ & (0.01624) \\
\hline & & {$[0.021]$} & [0.03152] & [0.004] & [0.02045] & [0.02062] & [0.02077] \\
\hline & 3000 & 0.01059 & 0.19945 & 0.01026 & 0.09187 & 0.89782 & 0.15056 \\
\hline & & $(0.00949)$ & $(0.01377)$ & $(0.00159)$ & $(0.00928)$ & $(0.0914)$ & $(0.01029)$ \\
\hline & & [0.01288] & {$[0.01753]$} & {$[0.00232]$} & [0.01533] & {$[0.01465]$} & [0.01379] \\
\hline
\end{tabular}

This table summarizes the estimates coefficients from the $\mathrm{AR}(1)-\mathrm{BL}-\mathrm{GARCH}(1,1)$ model with the true value set of parameters $\left\{\alpha_{0}, \alpha_{1}, a_{0}, a_{1}, b_{1}, c_{1}\right\}=\{0.01,0.2,0.01,0.09,0.9,0.15\}$. MAE - Mean Absolute Errors, RMSE - root mean square error for the $\mathrm{AR}(1)$-BL-GARCH(1,1). Monte Carlo simulations are computed with 1000 replications. Each replication gives a sample size $n=500,1000,2000$ and 3000 of observations. 


\section{Empirical study}

The daily continuously compounded returns of the S\&P 500 stock market index are used for the empirical study in this paper to gauge the effectiveness of the BLGARCH-type model with Normal, Student-t and GED innovations. In particular, we analyze the period from March 01, 1999 through January 31, 2001, which yields $n=487$ daily observations, excluding public holidays. The sample closely corresponds to the data used by Storti and Vitale (2003b). Table 6 gives the summary statistics of the S\&P $500 \log$ returns for the full sample. The mean and the standard deviation are quite small, while the estimated measure of skewness is significantly positive, indicating that the S\&P 500 has non-symmetric returns. The kurtosis is a little higher than that of a Normal distribution which is 3, suggesting that fat-tailed distributions could better describe the unconditional distribution of the data. The results of the non-Normality test agree with prior literature using financial data, that is, a leptokurtic distribution is found for the S\&P 500 log return data. The Box-Pierce $Q$-tests of up to twenty-fourth order serial correlation for the levels and squares of the mean-corrected S\&P $500 \log$ returns were performed. $Q(24)$ and $Q^{2}(24)$ are significant for both the return and squared return series. The diagnostics suggest that a GARCH-class model would be appropriate, along with an error distribution that allows for greater kurtosis than the Normal distribution.

Table 6

Statistics of daily log returns of the S\&P 500 stock market index.

\begin{tabular}{llll}
\hline Number of observations & 487 & Skewness & 0.03708 \\
Mean & 0.0002055 & Kurtosis & 4.4643 \\
Standard deviation & 0.01283 & Jarque Bera test & 43.3524 \\
Minimum & -0.06004 & $Q(24)$ & 39.2169 \\
Maximum & 0.04888 & $Q^{2}(24)$ & 45.7208 \\
\hline
\end{tabular}

The Jarque Bera test critical value at significance level of 5\% is 5.85423. $Q$ and $Q^{2}$ are the Box Pierce statistics for the levels and squared of the S\&P $500 \log$ returns respectively, using 24 lags. The critical value at level of $5 \%$ is 36.4150 .

Figure 5 gives, the time plot of the data while Figure 6 shows that the returns distribution also exhibits fat-tails confirming the results in Table 6.

Table 7 presents the results of maximum likelihood estimation of the parameters of the model under candidate the various assumptions on innovations, with the standard errors computed using the Hessian matrix ${ }^{4}$. The parameter estimates from

4 The time for convergence of the algorithm under specific distribution is approximately 5 seconds. 


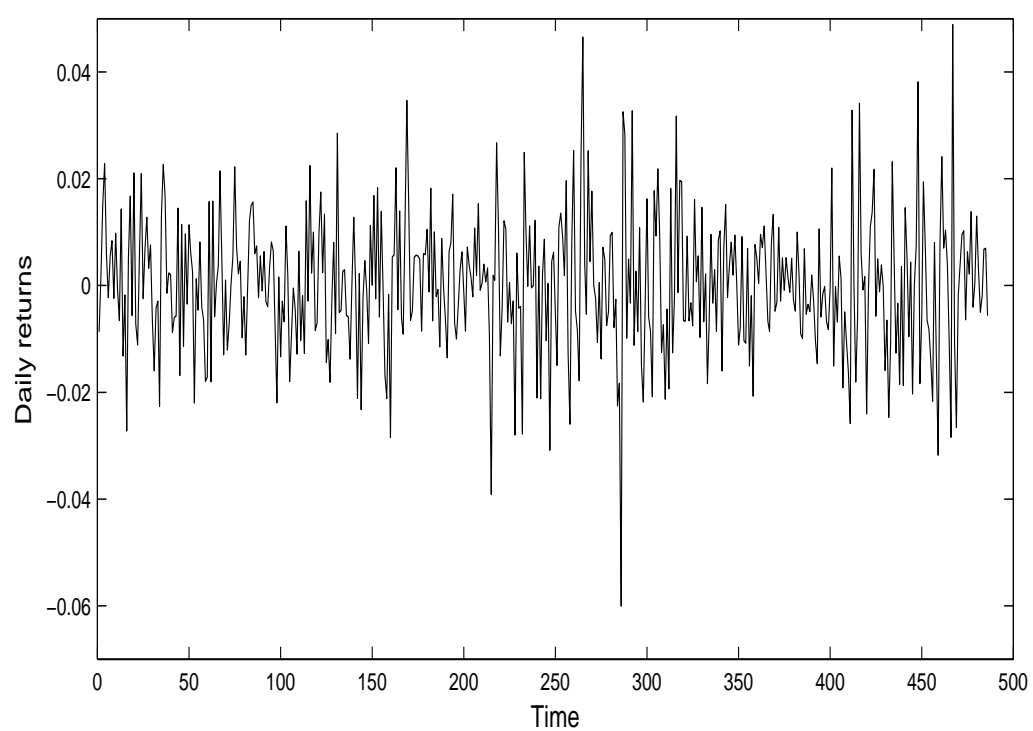

Fig. 5. S\&P 500 daily returns 03/01/99-01/31/01

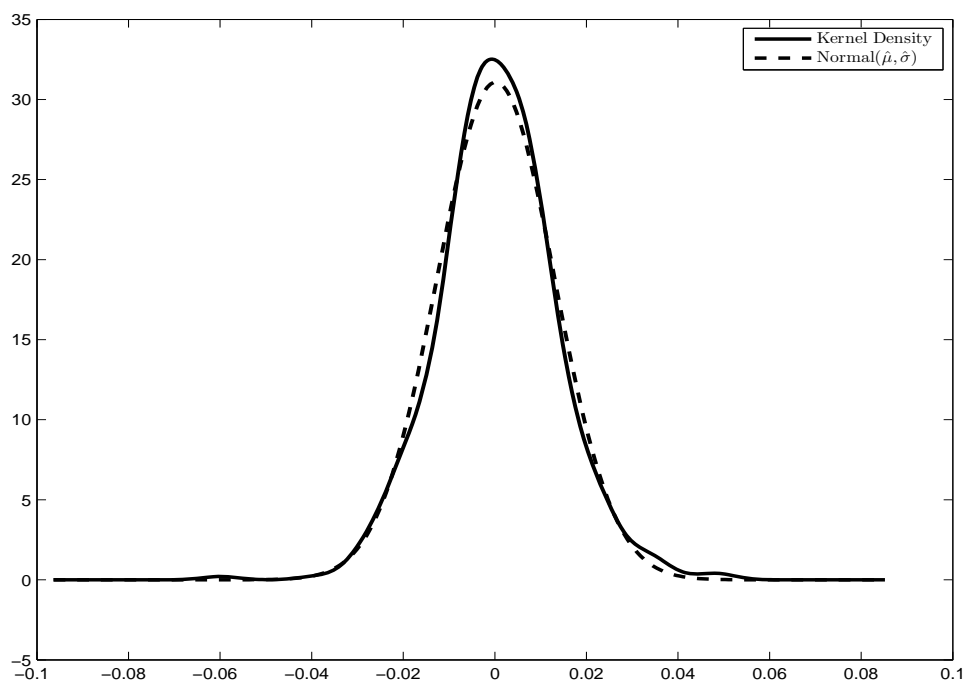

Fig. 6. Non-parametric density of S\&P 500 daily returns and probability density function of the normal distribution, in dotted line

the GARCH model with Normal, Student-t and GED errors are also provided in this table. In order to compare objectively, various goodness-of-fit statistics are used. The diagnostics, summarized in Table 7, are the log-likelihood function at its maximum, the Akaike Information Criterion (AIC) and the Bayesian Information Crietrion (BIC). We further report the values of the Box-Pierce $(Q)$ statistics for the standardized and squared standardized residuals with in parentheses, the corresponding p-values as a check of the empirical validity of the models. It is clear from Table 7 that estimate $\hat{a}_{1}$ and $\hat{b}_{1}$ in the $\operatorname{GARCH}(1,1)$ are significant at the $5 \%$ level 
with the volatility coefficient greater in magnitude. Hence the hypothesis of constant variance can be rejected, at least within sample. Furthermore, the stationarity condition is satisfied for the three distributions, as $\hat{a}_{1}+\hat{b}_{1}<1$ at the maximum of the respective log-likelihood functions.

From results obtained on estimation of the $\operatorname{BL}-\mathrm{GARCH}(1,1)$ model, we remark, once again, that the innovation and volatility spillovers are significantly different from zero. Further, the estimated asymmetric volatility response $\left(\hat{c}_{1}\right)$ is negative and significant for all models confirming the usual expectation in stock markets where downward movements (falling returns) are followed by higher volatility than upward movements (increasing returns). In all cases, the tails parameter estimate is strongly significant far two for the GED distribution while it is very large through insignificant for the Student-t distribution but $1 / v$ is also different to zero. However, the results confirm the empirical findings by Storti and Vitale (2003a), in that the kurtosis strongly depends to the leverage-effect response parameter.

The results for $Q$-statistics shown in Table 7 are not significant up to order 12 and also order 24, which indicates that the $\operatorname{BL}-\operatorname{GARCH}(1,1)$ as well as the $\operatorname{GARCH}(1,1)$ process are appropriate to model the conditional variance of the S\&P 500 logreturns. However, the goodness-of-fit statistics as well as the residuals diagnostics indicate that the BL-GARCH performs better in describing the conditional variance of the S\&P 500 returns. Moreover, the possible usefulness of using fat-tailed innovations for the BL-GARCH model seems to be confirmed by the log-likelihood values and the AIC.

\section{Conclusion}

This study obtains exact maximum likelihood estimates of a BL-GARCH process with conditionally elliptical distributions. The small-sample properties indicate that the approach can yield asymptotically efficient estimates. In addition, these results strongly suggest that the maximum likelihood estimation inference procedure can be used to estimate the parameters of the BL-GARCH model, even in samples as small as 100 observations. Further, as the simulation experiments show, one advantage of the maximum likelihood estimator procedure, proposed in this paper, compared to the method used by Storti and Vitale (2003b), is that it could simultaneously estimate the parameters of the BL-GARCH when the conditional mean is assumed non-constant. Further, the empirical results reveal that the BL-GARCH$\mathrm{t}(1,1)$, i.e a BL-GARCH model with conditional errors that are $\mathrm{t}$-distributed, fits the data best. 
Table 7

Conditional variance model estimates for the S\&P 500 return

\begin{tabular}{|c|c|c|c|c|c|c|}
\hline \multirow[b]{2}{*}{ Parameters } & \multicolumn{2}{|c|}{ GARCH model } & \multicolumn{4}{|c|}{ BL-GARCH model } \\
\hline & Normal & Student-t & GED & Normal & Student-t & GED \\
\hline \multirow[t]{2}{*}{$\hat{a_{0}}$} & 0.00000715 & 0.000006361 & 0.000006108 & 0.000011394 & 0.000009243 & 0.00001057 \\
\hline & $\left(0.2810410^{-10}\right)$ & $\left(0.2058610^{-10}\right)$ & $\left(0.2014410^{-10}\right)$ & $\left(0.1477510^{-10}\right)$ & $\left(0.1349610^{-10}\right)$ & $\left(0.1542410^{-10}\right)$ \\
\hline \multirow[t]{2}{*}{$\hat{a}_{1}$} & 0.0568184 & 0.0504452 & 0.0499355 & 0.060119 & 0.0513906 & 0.0559965 \\
\hline & $(0.0007625)$ & $(0.0006168)$ & $(0.0006111)$ & $(0.0006226)$ & $(0.0005249)$ & $(0.0006235)$ \\
\hline \multirow[t]{2}{*}{$\hat{b_{1}}$} & 0.900428 & 0.911250 & 0.913097 & 0.880531 & 0.900687 & 0.888784 \\
\hline & $(0.0026979)$ & $(0.0017480)$ & $(0.0018101)$ & $(0.0013771)$ & $(0.0011259)$ & $(0.0013873)$ \\
\hline \multirow[t]{2}{*}{$\hat{c_{1}}$} & - & - & - & -0.271323 & -0.249673 & -0.261943 \\
\hline & $(-)$ & $(-)$ & $(-)$ & $(0.0027800)$ & $(0.0030085)$ & $(0.0031051)$ \\
\hline \multirow[t]{2}{*}{$\hat{v}$} & - & 8.676471 & 1.514841 & - & 14.943269 & 1.741412 \\
\hline & $(-)$ & $(9.929491)$ & $(0.017712)$ & $(-)$ & $(67.446372)$ & $(0.024844)$ \\
\hline \multicolumn{7}{|c|}{ Goodness-of-fit statistics } \\
\hline Log-lik & 1435.91706 & 1441.94452 & 1441.04650 & 1456.47965 & 1458.63396 & 1457.65676 \\
\hline AIC & -2865.83441 & -2875.88904 & -2874.09300 & -2904.95930 & -2907.26792 & -2905.31353 \\
\hline \multicolumn{7}{|c|}{ Diagnostics } \\
\hline \multirow[t]{2}{*}{$Q(12)$} & 15.892571 & 16.018537 & 16.010225 & 15.876516 & 15.739415 & 15.833319 \\
\hline & $(0.196206)$ & $(0.190388)$ & $(0.190768)$ & $(0.196958)$ & $(0.203465)$ & $(0.198990)$ \\
\hline \multirow[t]{2}{*}{$Q(24)$} & 32.082112 & 32.206641 & 32.159427 & 32.314595 & 32.501633 & 32.417392 \\
\hline & $(0.124969)$ & $(0.121951)$ & $(0.123088)$ & $(0.119381)$ & $(0.115033)$ & $(0.116975)$ \\
\hline \multirow[t]{2}{*}{$Q^{2}(12)$} & 4.682633 & 4.589348 & 4.566436 & 4.477202 & 4.455119 & 4.485078 \\
\hline & $(0.967748)$ & (0.970309) & $(0.970918)$ & $(0.973208)$ & $(0.973756)$ & $(0.973011)$ \\
\hline \multirow[t]{2}{*}{$Q^{2}(24)$} & 19.326186 & 19.483667 & 19.377018 & 22.177684 & 22.542585 & 22.475412 \\
\hline & $(0.734378)$ & $(0.725728)$ & $(0.731595)$ & $(0.568662)$ & $(0.546916)$ & $(0.550914)$ \\
\hline
\end{tabular}

This table provides the estimated coefficients, standard errors for the conditional standard deviation equation for the S\&P $500 \log$ returns index market. $\hat{a}_{0}$ is the constant in the conditional standard deviation equation, $\hat{a}_{1}$ is the ARCH coefficient, $\hat{b}_{1}$ is the GARCH coefficient, $\hat{c_{1}}$ is the leverage effect, $\hat{v}$ is the degrees of freedom. Log-lik is the maximized log likelihood. AIC is the Akaike Information Citerion and BIC the Bayesian Information Criterion. $Q$ and $Q^{2}$ are the Box Pierce statistics for the standardized and squared standardized residuals respectively, using 12 and 24 lags with p-values in square brackets. The critical values at significant level of $5 \%$ are 21.026069 and 36.415028 respectively.

\section{References}

[1] A. Azzalini, and A. Capitanio, (2003) Distributions generated by perturbation of symmetry with emphasis on a multivariate skew t student distribution, Journal of the Royal Statistical Society Series B 65: 579-602.

[2] R.T. Baillie, and T. Bollerslev, Intra-day and inter-market volatility in foreign 
exchange rates, Review of Economic Studies 58 (1191), pp. 565-585.

[3] N.H. Bingham, and R. Kiesel, (2002) Semi-parametric modelling in finance: theoretical foundations, Quantitative Finance, 2: 368-385.

[4] F. Black, (1976) Studies in stock price volatility changes, in Proceedings of 1976 Meeting of Business and Economics Statistics Section, American Statistical Association, 177-181.

[5] T. Bollerslev, (1986) Generalized Autoregressive Conditional Heteroskedasticity, Journal of Econometrics, 31: 307-327.

[6] T. Bollerslev, (1987) A conditional heteroscedastic time series model for speculation prices and rates of return, Review of Economics and Statistics, 69: 542547.

[7] Z. Ding, C.W.Z. Granger, and R.F. Engle, (1993) A long memory property of stock market returns and a new model, Journal of Empirical Finance, 1: 83-106

[8] R.F. Engle, (1982) Autoregressive conditional heteroskedasticity with estimates of the variance of the U.K. inflation, Econometrica, 50: 987-1008.

[9] L. Giraitis, R. Leipus, and D. Surgailis, (2007) Recent advances in ARCH modelling, in G. Teyssière and A.P. Kirman (Eds.), Long Memory in Economics, Springer, Berlin.

[10] C. Gouriéroux, (1997) ARCH model and financial application, Springer, New York.

[11] B. Hansen, (1994) Autoregressive conditional density estimation, International Economic Review, 35: 705-730.

[12] M.B. Jones, and M.J. Faddy, (2003) A skew extension of the t distribution with applications, Journal of the Royal Statistical Society series B, 65: 159-174

[13] M. McAleer, (2005) Automated inference and learning in modeling financial volatility, Econometric Theory, 21: 232-261.

[14] D. B. Nelson, (1991) Conditional heteroskedasticity in asset returns: A new approach, Econometrica, 59: 347-370.

[15] A. Patton,(2004) On the out-of-sample importance of skewness and asymmetric dependence for asset allocation, Journal of Financial Econometrics, 2: 130-168.

[16] A. Peiró, (1999) Skewness in financial returns, Journal of Banking and Finance, 23: 847-862.

[17] G. Storti, C.Vitale, (2003a) BL-GARCH models and asymmetries in volatility, Statistical Methods and Applications, 12: 19-40.

[18] G. Storti, C.Vitale, (2003b) Likelihood inference in BL-GARCH models, Computational Statistics, 18: 387-400.

[19] R.S. Tsay, (2002) Analysis of financial time series, Wiley, New York.

[20] E. Zivot, and J. Wang, (2005) Modeling financial time series with SPLUS, Springer Berlin Heidelberg, New York. 